The above discussion must be viewed with caution, as a second interpretation of the findings may be made on the basis of the topography of the visual cortex. Due to the spatial representation of the retina in the visual cortex, we may assume that the upper- and lower-field responses obtained in this study originated at distinctly different areas of the striate cortex within the calcarine fissure. Thus the potentials recorded between the scalp and earlobe may have been affected by differences in the volume conduction characteristics of the brain in relation to the loci of origin of the evoked responses. Perhaps greater attenuation occurred in the volume conductor in relation to the site of origin of the upper-field responses and that these responses at their site of origin were actually equal to or greater than the lower-field responses occurring in the calcarine fissure. Jeffreys (1968) has reported some electrode location data which suggests that the topographical factor may be important. Work is under way in our laboratory to test further this topographical hypothesis.

Even if the topographical explanation should have some validity in accounting for upper-lower field differences, it seems an unlikely candidate for explaining the effects of check size on evoked potential amplitude. There is no reason to assume that the effects are due to differences in the origin of the evoked response within the calcarine fissure, since exactly the same retinal area within each field is stimulated with each checkerboard stimulus display. It seems equally unlikely that the shift in maximal response amplitude from larger to smaller checks as the stimulus display is moved from the lower to the upper field (Fig. 2) is due to differences in the loci of origin of the responses.

The effects of check size may be more plausibly explained in terms of differences in the size of the centers of retinal receptive fields located in the retinal areas projecting respectively to the upper and lower visual fields. Harter \& White (1970) have built a rather strong case for the possibility that cortical evoked responses may be related to the size of receptive field centers, as reflected in the single unit responses of animals. Recently Harter (in press) obtained an interaction between check size and retinal eccentricity which was predicted from the hypothesis that evoked potentials and size of receptive field centers are related.

Assuming that the receptive field hypothesis has validity, the results depicted in Fig. 2 suggest that the receptive field centers located in that part of the retina stimulated from within the lower field have an average size of approximately $40 \mathrm{~min}$, whereas those which project to the upper field subtend about 10 min of visual angle. Why the retina projecting to the upper field should have smaller receptive-field centers than the area projecting to the lower field is not entirely clear. One may speculate that flying or rapidly moving objects appearing in the upper field must be detected while relatively far away if the organism is to respond appropriately to them. Thus, the part of the visual system projecting to the upper field may be particularly attuned to "specks in the sky" subtending less than $30 \mathrm{~min}$ of visual angle. On the other hand, ground objects which are sufficiently close to the organism to be potentially threatening generally may subtend somewhat larger visual angles and therefore that part of the visual system projecting to the lower field developed a relatively high degree of reactivity to objects subtending 30 or more minutes of visual angle.

\section{REFERENCES}

EASON, R. G., ODEN, D., \& WHITE, C. T. $V$ isually evoked cortical potentials and reaction time in relation to site of retinal stimulation. EEG Clinical Neurophysiology, 1967, 22, 313-324.

EASON, R. G., \& WHITE, C. T. Averaged occipital responses to stimulation of sites in the nasal and temporal halves of the retina. Psychonomic Science, 1967, 7, 309-310.

EASON, R. G., WHITE, C. T., \& ODEN, D. Averaged occipital responses to stimulation of sites in the upper and lower halves of the retina. Perception \& Psychophysics, 1967, 2, 423-425.

HARTER, M. R. Evoked cortical responses to checkerboard patterns: Effect of check-size as a function of retinal eccentricity. Vision Research, in press.

HARTER, M. R., \& SUITT, C. D. Visually-evoked cortical responses and pattern vision in the infant: A longitudinal study. Psychonomic Science, 1970, 18, 235-237.

HARTER, M. R., \& WHITE, C. T. Effects of contour sharpness and check-size on visually evoked cortical potentials. Vision Research, $1968,8,701-711$

HARTER, M. R., \& WHITE, C. T. Evoked cortical responses to checkerboard patterns: Effect of check-size as a function of visual acuity. EEG Clinical Neurophysiology, 1970, $28,48-54$

JEFFREYS, D. A. Separable components of human evoked responses to spatially patterned visual fields. EEG Clinical Neurophysiology, 1968, 24, 596. (Abstract)

JOHN, E. R., HERRINGTON, R. N., \& SUTTON, S. Effects of visual form on the evoked response. Science, 1967, 155, 1436-1442.

MackAY, D. M. Evoked brain potentials as indicators of sensory information processing. Neurosciences Research Program Bulletin, $1969,7,211-216$.

RIETVELD, W. J., TORDOIR, W. E., HAGENOUW, J. R., LUBBERS, J. A., \& SPOOR, Th. A. C. Visual evoked responses to blank and to checkerboard patterned flashes. Acta Physiol. Pharmacol. Neerl., 1967, 14, 259-285.

SPEHLMANN, R. The averaged electrical responses to diffuse and to patterned light in the human. EEG Clinical Neurophysiology, $1965,19,560-569$.

\title{
The orienting reflex to changes in a conceptual stimulus dimension*
}

\section{R. M. YAREMKO, MARJORIE W. BLAIR, and BRUCE T. LECKART San Diego State College, San Diego, Calif. 92115}

The galvanic skin response (GSR) component of the orienting reflex was recorded for four groups of $12 \mathrm{Ss}$ each. They were given 10 habituation trials to black on white numeric stimuli presented in seriatum. On Trial 11 an out-of-sequence test stimulus (TS) was delivered and was followed by one additional in-sequence number. The TS was either \pm 1 or \pm 11 places out of sequence. It was found that GSR magnitude to the TS was primarily a function of the amount of disparity provided by the TS, irrespective of the direction of that disparity. Dishabituation to the subsequent in-sequence stimulus was not, however, clearly present. The results were related to Sokolov's neuronal model of habituation and to previous research.

According to Sokolov (1963), magnitude of the orienting reflex (OR) is primarily a function of the amount of

* Supported in part by a grant to the first author from the San Diego State College Foundation. Requests for reprints should be addressed to R. M. Yaremko, Department of Psychology, San Diego State College, San Diego, California 92115 . change in the parameters of ambient stimulation when directional dynamogenic effects are not operative. Much support for this contention has been reported (e.g., Kimmel, 1960; Zimny \& Schwabe, 1965; Yaremko, 1969).

Other recent investigations (Unger, 1964: Zimny, Pawlick, \& Saur, 1969) indicate that the stimulus parameters of 
Table 1

Mean GSR Magnitudes $(\Delta \log C \times 1,000)$ for Each Direction and Amount of Change Condition

\begin{tabular}{ccc}
\hline $\begin{array}{c}\text { Direction } \\
\text { of } \\
\text { Change }\end{array}$ & \multicolumn{2}{c}{ Amount of Change } \\
\cline { 2 - 3 } & 1 & 11 \\
\hline- & 7.8 & 19.7 \\
+ & 13.2 & 25.4 \\
\hline
\end{tabular}

habituation described by Sokolov may extend beyond simple physical dimensions, such as stimulus intensity, frequency, duration, signal-to-noise ratio, etc. In these studies the "conceptual" dimension provided by delivering numbers in seriatum was varied. In sum, these studies demonstrated that OR recovery was effected by a change (the delivery of an out-of-sequence number) in the developing conceptual stimulus dimension.

The present study was designed to test Sokolov's predictions regarding OR recovery and dishabituation as a function of direction and amount of change in the conceptual stimulus dimension. Specifically, it was predicted that, after $S$ was exposed to a sequence of in seriatum numbers, OR recovery, as indexed by the galvanic skin response (GSR), would be a function of amount, rather than direction in which an out-of-sequence test stimulus (TS) was varied.

\section{SUBJECTS AND APPARATUS}

Thirty-three female and 15 male undergraduates at San Diego State College volunteered in order to meet a class requirement. They were assigned randomly to one of four experimental conditions upon reporting to the laboratory.

Data were collected from the $S$ seated in an IAC Model 401-A chamber containing a cushoined chair. The GSR was picked up from the palm and back of S's right hand by $14-\mathrm{mm}$-diam zinc-zinc sulphate electrodes in Lucite cups filled with Redux electrode jelly. The response was amplified and recorded by a Grass Model 5-D polygraph at a paper speed of $1.5 \mathrm{~mm} / \mathrm{sec}$. Numbers were projected on a translucent Plexiglas screen $(35 \times 55 \mathrm{~cm})$ mounted on the chamber window in front of $S$ by a Kodak Model 850 projector. A light-tight tunnel extended from the projector lens to the screen. Numbers were black on white and approximately $20 \mathrm{~cm}$ high when projected. The distance between $\mathrm{S}$ and the screen was approximately $1.0 \mathrm{~m}$.

\section{PROCEDURE}

The S's hand was cleaned with acetone and the electrodes were attached. During this time $S$ was told informally that the study was designed to investigate simple physiological responses to external stimulation. The $S$ was cautioned to remain alert, avoid excessive movement, and simply to view whatever appeared on the screen in front of him. These instructions were repeated through an intercom after $S$ was seated in the chamber. Following a 5 -min initial rest period, the numbers 10 through 19 were delivered serially to S. On the eleventh trial (when 20 would have been presented) a TS of $31,21,19$, or 9 was delivered. Thus, the TS was either \pm 1 or \pm 11 places out of sequence. On Trial 12 the series was resumed; it terminated with the number 20 . The $S$ was then informally debriefed. Stimulus duration was always $10 \mathrm{sec}$, and the intertrial interval varied between 30 and $60 \mathrm{sec}$, with a mean of 45 sec.

\section{RESULTS}

An OR was defined as the first GSR within 1.5 to $6.0 \mathrm{sec}$ of stimulus onset. ${ }^{1}$ The response was transformed into log-conductance units by the formula: $\log$ (resistance before/resistance after). The measure of OR recovery to the TS was S's response to the TS minus his response to the last in sequence stimulus (Trial 10). These data are presented in Table 1. Inspection of the table suggests that large-change TSs tended to evoke larger responses than small-change TSs in general, but that there was some tendency for negative direction TSs to evoke smaller responses.

Analysis of variance partially supported these observations. The amount of change variable was significant $(p<.025)$, but the direction of change variable and the Amount by Direction interaction did not approach significance. Duncan's multiple range test indicated that the positive and negative large-change groups each differed from their respective small-change groups $(\mathrm{p}<.05$ each) and that the large positive change produced significantly greater responding than did the small negative change $(\mathrm{p}<.01)$. No other group differences were significant.

The difference between responding on Trial 10 and Trial 12 was computed as a measure of the dishabituating capacity of the TS when the series was resumed. Although some Ss displayed observable OR dishabituation, no reliable increments or group differences were uncovered.

\section{DISCUSSION}

These results appear to support Sokolov's hypotheses concerning the relative roles of direction and amount of change in stimulus parameters on evocation of the OR, since it was convincingly demonstrated that $O R$ magnitude was primarily a function of the amount of discrepancy between the expected stimulus (the number 20) and the observed TS. Ancillary predictions based on Sokolov's description of the $\mathrm{OR}$, however, lead to the expectation that dishabituation of the OR to subsequent in-series stimuli would occur as a function of the amount of disparity present in the test trial. Although this finding was reported by Zimny \& Schwabe (1965) for tonal stimuli, it was not clearly present in this study or in Zimny, Pawlick, \& Saur's (1969) report.

One somewhat bothersome aspect of the present study deserves comment. While the number 19 would appear to qualify as a novel TS by virtue of the fact that $S$ presumably "expected" the number 20 on the test trial, it does differ from the other TSs in that the same stimulus was presented on the immediately preceding trial. Such repetition, of course, would lead to the prediction that response magnitude to 19 would be smaller than response magnitudes to the other three TSs. (Incidentally, this was observed in the present study.) Thus, the somewhat ambiguous status of this stimulus demands that comparisons between this study and those involving manipulations of stimulus dimensions in which all TSs are novel (e.g., Kimmel, 1960) should be approached with caution.

Basically, though, these findings confirm and extend those of Unger (1964) and Zimny et al (1969) and certainly indicate that at least some of the parameters of the OR transcend the level of simple physical stimuli. In this respect the present study also suggests that $O R$ and related research involving novelty manipulations in semantic, verbal, and other conceptual dimensions might profit from rigorous investigations of quantitative and directional changes in the stimulus.

$$
\text { REFERENCES }
$$

KIMMEL, H. D. The relationship between direction and amount of stimulus change and amount of perceptual disparity response. Journal of Experimental Psychology, 1960, $59,68-72$

SOKOLOV, E. N. Perception and the conditioned reflex. New York: Pergamon Press, 1963.

UNGER, S. M. Habituation of the vasoconstrictive orienting reaction. Journal of Experimental Psychology, 1964, 67; 11-18

YAREMKO, R. M. Effects of direction and amount of change in stimulus intensity on magnitude of the orienting reflex and frequency of signal detection. Unpublished PhD dissertation, Ohio University, 1969.

ZIMNY, G. H., PAWLICK, G. F., \& SAUR, D. P. Effects of stimulus order and novelty on orienting responses. Psychophysiology, 1969, $6,166-173$.

ZIMNY, G. H., \& SCHWABE, L. W. Stimulus change and habituation of the orienting reflex. Psychophysiology, 1965, 2, 103-115. NOTE

1. The conventional $4.0-$ to $4.5-\mathrm{sec}$ maximum latency criterion was liberalized when, as a result of S's debriefing, it was discovered that Ss were not always attending to the screen at the moment of stimulus onset. 